\title{
Improving Maps of Physical and Virtual Radio Transmitters
}

\author{
Markus Ulmschneider, German Aerospace Center (DLR) \\ Christian Gentner, German Aerospace Center (DLR)
}

\begin{abstract}
BIOGRAPHIES
Markus Ulmschneider studied communications and computer engineering at the University of Ulm, Germany, from where he received his Bachelor's degree in 2011 and his Master's degree in 2014. In 2014, he joined the Institute of Communications and Navigation of the German Aerospace Center (DLR), Germany, where he is part of the scientific staff of the Mobile Radio Transmission group. His main research interests include multipath assisted positioning as well as multi-sensor localization and tracking techniques.
\end{abstract}

Christian Gentner studied electrical engineering at the University of Applied Science in Ravensburg, with the main topic communication technology and received his Dipl.-Ing. (BA) degree in 2006. During this study he received practical experiences at Rohde \& Schwarz in Munich. He continued his study at the University of Ulm in 2006. He received the M.Sc. and Dr.-Ing. (PhD) degree in 2009 and 2018 respectively, both from the University of Ulm. Since 2009, he is working at the Institute of Communications and Navigation of the German Aerospace Center (DLR). His current research focuses on multipath assisted positioning.

\begin{abstract}
In multipath assisted positioning, each multipath component arriving at a receiver is regarded as being sent by a virtual transmitter in a line-of-sight condition. These virtual transmitters can be used for localizing a user. In the general case, their locations are unknown. With simultaneous localization and mapping (SLAM), the locations of physical and virtual transmitters can be estimated jointly with the location of a user. However, the convergence time can be high. In scenarios such as malls or museums, multiple users travel through the same scenario on different trajectories. Different users can cooperate by exchanging maps of physical and virtual transmitters in order to decrease the convergence time and increase the positioning performance. Though, each user is in its own local coordinate system. When a map of transmitters is handed from one user to another, the relative rotation and translation of the coordinate systems of the two users need to be estimated based on the transmitters in the two users' maps. Within this paper, we propose an algorithm to merge and update an obtained transmitter map with the transmitters estimated by the current user. We evaluate our proposed method by simulations in an indoor mall. In a crowdsourcing based approach, maps are handed from one user to another, updated, and handed to the next user. Our evaluations show that exchanging transmitter maps improves the positioning performance, although each user is in its own local coordinate system not knowing the parameters relating its own coordinate system to the ones of obtained transmitter maps.
\end{abstract}

\section{INTRODUCTION}

Multipath assisted positioning exploits the spatial information about multipath components (MPCs) of signals of opportunity (SoOs). In our approach called Channel-SLAM [1], each multipath component of a terrestrial radio signal corresponds to a virtual transmitter that can be used for localization of a user. Thus, each of the detected signal components corresponds to a physical (line-of-sight component) or virtual (MPC) transmitter, and Channel-SLAM does not differentiate between physical and virtual transmitters. There are approaches [2], [3] that assume the location of the physical transmitter and the geometry of the scenario to be known, and hence the locations of the virtual transmitters can be calculated in advance. Channel-SLAM assumes no prior knowledge about the scenario or the location of any transmitter. Thus, we localize both the user and the transmitters with simultaneous localization and mapping (SLAM) [4]. In SLAM terms, the user is localized simultaneously with mapping the transmitters.

Unlike standard radio-based localization approaches, multipath assisted positioning considers multipath propagation not as harmful, but beneficial for localization. In areas with a rich multipath propagation, multipath assisted positioning can support or replace other positioning systems such as global navigation satellite systems (GNSSs). In particular, in urban scenarios or indoors, where the positioning performance of GNSSs is degraded or no positioning solution can be obtained at all, and multipath propagation degrades the performance of standard radio-based approaches, multipath assisted positioning can exploit its full potential. In these scenarios, physical transmitters of SoOs could be Wi-Fi routers or cell towers, for example. 


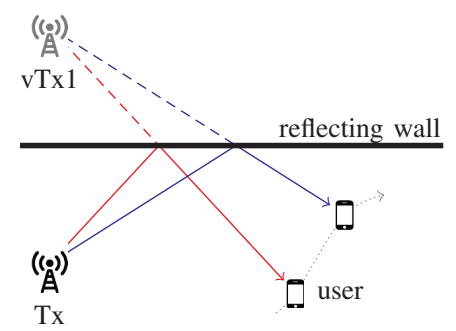

Fig. 1. The signal from the physical transmitter Tx is reflected at the wall and arrives at the user as a MPC. The reflected signal is interpreted as a LoS signal from the virtual transmitter vTx1. The location of vTx1 is the location of Tx mirrored at the reflecting wall.

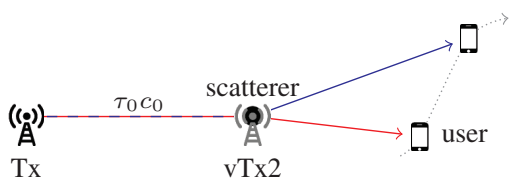

Fig. 2. The signal from the physical transmitter Tx is scattered at a point scatterer and arrives at the user as a MPC. The scattered signal is regarded as a LoS signal from the virtual transmitter vTx2, which is located at the scatterer location. The virtual transmitter has an additional delay offset $\tau_{0}$ towards the physical transmitter, which is the Euclidean distance between the transmitters divided by the speed of light $c_{0}$.

In shopping malls or museums, for example, there is typically a high fluctuation of users. Hence, maps of transmitters can be exchanged among users and serve as prior information increasing the positioning performance. With crowdsourcing, transmitter maps can be created and continuously updated by the information from measurements of users without the necessity of additional infrastructure.

Though, Channel-SLAM is only a relative localization approach. Each user is within its own local coordinate system. When maps are exchanged, they are in different coordinate systems with an unknown relative rotation and translation. Based on the transmitters estimated by the user, i.e., the transmitters in the user map, and the transmitters in a map received from a different user, i.e., a prior map, the relative rotation and translation between the coordinate systems need to be estimated before the information in a prior map can be exploited.

In a previous paper [5], we have studied how to estimate the rotation and translation among two transmitter maps, where we exploit relative distances among transmitters within one map. We have shown that the positioning mean absolute error (MAE) of one single user is decreased significantly once the rotation and translation between the coordinate systems of the user and a prior map are estimated.

Within this paper, we will investigate how multiple users can consequently improve a prior map created by one user in an indoor scenario. The users walk through the scenario on different paths. The first user has no prior information on any transmitter, and creates an initial transmitter map. This map is handed from one user to another, and each of these users both exploits and improves the map under the constraint that the map is in a coordinate system of unknown rotation and translation to the current user's coordinate system. Thus, the rotation and translation parameters have to be estimated before the information in the prior map can be used and updated. Finally, the transmitters whose states are estimated by the user and the transmitters from the prior map have to be merged into a new map that is shared with other users. Each transmitter's state probability density function (PDF) is approximated by a particle cloud. To reduce the communication load, the new map needs to be stored efficiently.

We have performed simulations in an indoor mall to evaluate our approach. The results show that the user MAE can be decreased if maps are shared among users, although these maps are in different coordinate systems.

\section{PRINCIPLES}

\section{Multipath Assisted Positioning}

The fundamental idea of multipath assisted positioning is presented in Fig. 1 and Fig. 2. In Fig. 1, the physical transmitter Tx broadcasts a signal that is received by the user after being reflected at the wall. Though, the received signal component can be regarded as being transmitted by the virtual transmitter vTx 1 in a LoS condition. While the reflection point on the wall moves as the user moves, the location of vTx 1 is static. Indeed, the location of vTx1 is obtained by mirroring the physical transmitter location at the reflecting wall. The physical and virtual transmitters are inherently perfectly time synchronized.

A similar scenario is depicted in Fig. 2. The signal from the physical transmitter Tx is now scattered at a point scatterer. Again, the scattered signal can be regarded as being sent by the virtual transmitter vTx 2 in a LoS condition. Though, the 
physical transmitter and the virtual transmitter are not time synchronized in the case of scattering. The virtual transmitter has an additional delay offset $\tau_{0}$ towards the physical transmitter. The delay offset is the Euclidean distance between the transmitters divided by the speed of light, and it can be interpreted as a clock offset.

A generalization of the above cases to the case where the signal is reflected and/or scattered multiple times is straightforward $[1]$.

\section{Channel-SLAM}

Channel-SLAM assumes a linear and time-variant multipath channel between the static physical transmitter and the mobile user. The capability of resolving signal components at the receiver is crucial for exploiting them for positioning. In a first step, the Kalman enhanced super resolution tracking (KEST) estimator [6] is used to estimate the parameters of signal components of the received signal and track them over time. Depending on the available hardware, such parameters can be the time of arrival (ToA), power, or angle of arrival (AoA), for example. In our case, the ToA and AoA estimates from the KEST estimator at time instant $k$ are combined in the measurement vector

$$
\boldsymbol{z}_{k}=\left[\begin{array}{llllll}
d_{1, k} & \ldots & d_{N_{\mathrm{TX}}, k} & \theta_{1, k} & \ldots & \theta_{N_{\mathrm{TX}}, k}
\end{array}\right]^{T},
$$

where $d_{j, k}$ denotes the estimate for the ToA and $\theta_{j, k}$ the estimate for the AoA from the $j^{\text {th }}$ transmitter. The number of observable transmitters, $N_{\mathrm{TX}}$, is time-variant as it depends on the number of signal components estimated by KEST in each time instant. Nevertheless, the time instant index $k$ is omitted in $N_{\mathrm{TX}}$ for notational brevity.

The above estimates obtained from KEST are used in a second step in a SLAM scheme, where the states of the user and of the physical and virtual transmitters are estimated. In fact, Channel-SLAM does not differentiate between the physical and virtual transmitters, i.e., between the LoS component and MPCs: each signal component arriving at the receiver is regarded as a LoS signal from a transmitter, which can be physical or virtual. The user state vector $\boldsymbol{x}_{\mathrm{u}, k}$ at time instant $k$ comprises the user's location and velocity in two dimensions and is donated by

$$
\boldsymbol{x}_{\mathrm{u}, k}=\left[\begin{array}{llll}
x_{k} & y_{k} & v_{x, k} & v_{y, k}
\end{array}\right]^{T} .
$$

Since the two-dimensional transmitter locations are static and the transmitters have an unknown clock offset $\tau_{0}$, the state vector of the $j^{\text {th }}$ transmitter at time instant $k$ is given by

$$
\boldsymbol{x}_{\mathrm{TX}, k}^{<j>}=\left[\begin{array}{lll}
x_{\mathrm{TX}, k}^{<j>} & y_{\mathrm{TX}, k}^{<j>} & \tau_{0, k}^{<j>}
\end{array}\right]^{T} .
$$

Stacking the user state vector and the $N_{\mathrm{TX}}$ transmitters' state vectors together, the entire state vector at time instant $k$ is

$$
\boldsymbol{x}_{k}=\left[\begin{array}{llll}
\boldsymbol{x}_{\mathrm{u}, k}^{T} & \boldsymbol{x}_{\mathrm{TX}, k}^{<1>^{T}} \ldots \boldsymbol{x}_{\mathrm{TX}, k}^{<N_{\mathrm{TX}}>^{T}}
\end{array}\right]^{T} .
$$

We use a Bayesian recursive estimation scheme [7] to estimate the posterior likelihood $\mathrm{p}\left(\boldsymbol{x}_{0: k} \mid \boldsymbol{z}_{1: k}\right)$, where $\boldsymbol{x}_{0: k}$ denotes the state vector from time instants zero to $k$, and likewise $z_{1: k}$ denotes the stacked measurement vectors from time instants one to $k$. Due to the large number of dimensions in $\boldsymbol{x}_{k}$, we apply Rao-Blackwellization [8] to separate the user and the transmitters' state space. In addition, independence among the measurements for the different transmitters, i.e., among the estimates of the parameters for different signal components in KEST, is assumed. Then, the posterior likelihood $\mathrm{p}\left(\boldsymbol{x}_{0: k} \mid \boldsymbol{z}_{1: k}\right)$ can be factorized as

$$
\begin{aligned}
\mathrm{p}\left(\boldsymbol{x}_{0: k} \mid \boldsymbol{z}_{1: k}\right) & =\mathrm{p}\left(\boldsymbol{x}_{\mathrm{TX}, 0: k}, \boldsymbol{x}_{\mathrm{u}, 0: k} \mid \boldsymbol{z}_{1: k}\right) \\
& =\mathrm{p}\left(\boldsymbol{x}_{\mathrm{u}, 0: k} \mid \boldsymbol{z}_{1: k}\right) \mathrm{p}\left(\boldsymbol{x}_{\mathrm{TX}, 0: k} \mid \boldsymbol{x}_{\mathrm{u}, 0: k}, \boldsymbol{z}_{1: k}\right) \\
& =\mathrm{p}\left(\boldsymbol{x}_{\mathrm{u}, 0: k} \mid \boldsymbol{z}_{1: k}\right) \prod_{j=1}^{N_{\mathrm{TX}}} \mathrm{p}\left(\boldsymbol{x}_{\mathrm{TX}, 0: k}^{<j>} \mid \boldsymbol{x}_{\mathrm{u}, 0: k}, \boldsymbol{z}_{1: k}\right),
\end{aligned}
$$

where $\boldsymbol{x}_{\mathrm{TX}, 0: k}^{<j>}$ is the state vector of the $j^{\text {th }}$ transmitter from time instants zero to $k$.

To be able to cope with the non-linear measurement model, $\mathrm{p}\left(\boldsymbol{x}_{0: k} \mid \boldsymbol{z}_{1: k}\right)$ is estimated by a Rao-Blackwellized particle filter. The user state is estimated by a particle filter, and for each user particle, the transmitters states are estimated independently from each other. The state posterior distribution of the $j^{\text {th }}$ transmitter given the $i^{\text {th }}$ user particle's state vector $\boldsymbol{x}_{\mathrm{u}, k}^{<i>}$ is therefore

$$
\mathrm{p}\left(\boldsymbol{x}_{\mathrm{TX}, k}^{<i, j>} \mid \boldsymbol{z}_{1: k}, \boldsymbol{x}_{\mathrm{u}, k}^{<i>}\right)=\sum_{l=1}^{N_{p, \mathrm{Tx}}} w_{k}^{<i, j, l>} \delta\left(\boldsymbol{x}_{\mathrm{TX}, k}^{<i, j>}-\boldsymbol{x}_{\mathrm{TX}, k}^{<i, j, l>}\right),
$$




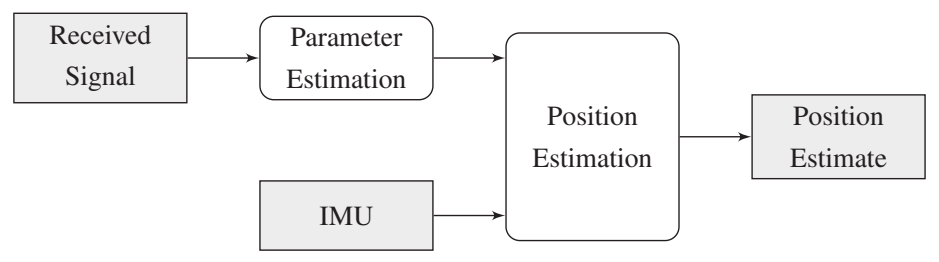

Fig. 3. Overview of the two steps of the Channel-SLAM algorithm.

where $N_{p, \mathrm{Tx}}$ is the number of transmitter particles, $\boldsymbol{x}_{\mathrm{TX}, k}^{<i, j, l>}$ is the $l^{\text {th }}$ transmitter particle's state vector and $w_{k}^{<i, j, l>}$ its associated weight. The Dirac delta distribution is donated by $\delta(\cdot)$. Although $N_{p, \text { Tx }}$ is time-variant and may differ for different user particles and different transmitters, we omit the time and transmitter indices in $N_{p \text {,Tx }}$.

Fig. 3 summarizes the two steps of Channel-SLAM. In the first step, KEST estimates the parameters of signal components based on the received signal, and in the second step, these estimates are used to simultaneously estimate the user location and map the physical and virtual transmitters. In the second step, additional sensors such as from an inertial measurement unit (IMU) can be used [1].

\section{Exchanging Transmitter Maps in Channel-SLAM}

When multiple users travel in the same scenario, they can cooperate by exchanging maps of physical and virtual transmitters. Though, Channel-SLAM is only a relative localization system, as the user location is determined relative to the observed transmitters. In general, there is no relation to a common coordinate system known, and each user creates its own local coordinate system. When a transmitter map is passed from one user to another, the coordinate systems of the two maps are related by an unknown rotation $\beta$ and translation $\gamma=\left[\begin{array}{ll}\bar{x} & \bar{y}\end{array}\right]$, which need to be estimated based on the transmitter state PDFs in the two maps. In addition, correspondences among transmitters in the two maps need to be found. This is to find which transmitters in the one map correspond to which transmitters in the other map. We define seeking the relative translation and rotation of the coordinate systems between two transmitter maps and the correspondences among transmitters in the two maps as map matching. If the relative translation and rotation parameters and the correspondences are found, the map match was successful. In [5], we have proposed a map matching scheme for Channel-SLAM which is briefly summarized in the following. The set of transmitter state PDFs estimated by the current user is denoted by the term user map, whereas a map obtained from a different user is called a prior map.

\section{Map Matching}

To find $\beta, \gamma$ and the correspondences, only transmitters in the two maps whose variances do not exceed a threshold $\delta_{\sigma}$ are used. The distance between two transmitters within one map is then defined as the Euclidean distance between the mean of the state PDFs of the two transmitters. While there is no information on the absolute locations of transmitters, the relative distances among transmitters in each map can be exploited. Based on these relative distances, the correspondences among transmitters are to be found. A set of correspondences among transmitters is a set of tuples $\left(j, \mathrm{P}_{r}\right)$ that contain one transmitter index $j$ from the user map and the corresponding transmitter index $\mathrm{P}_{r}$ from the prior map. For each possible set of correspondences of transmitters in the use map and prior map of a certain size, the squared differences between any two transmitter distances within each of the two maps are regarded. The correspondence set $\hat{\mathcal{C}}$ for which the sum of these squared differences is the smallest is chosen. Based on these correspondences, $\beta$ and $\gamma$ are obtained by a least square approach.

Map matching can be performed in parallel to the actual Channel-SLAM algorithm. The number of possible correspondences among transmitters in two maps can be very high resulting in a high computational complexity. Since the estimated transmitter state PDFs tend to change only little from one time instant to another, we try to find a map match only every $10^{\text {th }}$ time step, or when the variance of the state PDF of a transmitter in the user map falls below the threshold $\delta_{\sigma}$. A map match is successful if the map match error as calculated in [5] is below a threshold. Only then the prior map is transformed into the current user's coordinate system with the corresponding parameters $\beta$ and $\gamma$. Even when a map match was successful, we keep up map matching as the user moves. If a new map matching error is smaller than the error from a previous map match, the prior map is translated and rotated with the newly obtained parameters.

When a map match was successful, there are inherently associations obtained from the chosen set of correspondences $\hat{\mathcal{C}}$. If one element of this set is the tuple $\left(j, \mathrm{P}_{r}\right)$, where $j$ and $\mathrm{P}_{r}$ are the indices of the associated transmitters in the user map and the prior map, respectively, the new posterior density of the $j^{\text {th }}$ transmitter is

$$
\mathrm{p}\left(\tilde{\boldsymbol{x}}_{\mathrm{TX}, k}^{<i, j>} \mid \boldsymbol{z}_{1: k}, \boldsymbol{x}_{\mathrm{u}, k}^{<i>}\right)=\sum_{l=1}^{N_{p}^{<j>}} w_{k}^{<i, j, l>} \delta\left(\tilde{\boldsymbol{x}}_{\mathrm{TX}, k}^{<i, j>}-\boldsymbol{x}_{\mathrm{TX}, k}^{<i, j, l>}\right)+\sum_{l=1}^{N_{p, a}^{<\mathrm{P}_{r}>}} w_{a, k}^{<i, \mathrm{P}_{r}, l>} \delta\left(\tilde{\boldsymbol{x}}_{\mathrm{TX}, k}^{<i, j>}-\boldsymbol{x}_{\mathrm{TX}, a, k}^{<i, \mathrm{P}_{r}, l>}\right),
$$


where $\boldsymbol{x}_{\mathrm{TX}, a, k}^{<i, \mathrm{P}_{r}, l>}$ is the state of the $l^{\text {th }}$ particle of the $\mathrm{P}_{r}{ }^{\text {th }}$ transmitter in the prior map, $w_{a, k}^{<i, \mathrm{P}_{r}, l>}$ its associated weight, and $N_{p}^{<j>}$ and $N_{p, a}^{<\mathrm{P}_{r}>}$ denote the corresponding number of transmitter particles. Thus, the two transmitter state PDFs, i.e., particle clouds, are combined with equal ratio. The weights need to be normalized to one.

The transmitters in the prior map serve as prior information when the user initializes new transmitters. When KEST detects a new signal component, a new transmitter is initialized, and there are three possible cases: the new signal component corresponds to

1) a new transmitter that has not been observed before and is not in the prior map,

2) a transmitter that had been observed before, or

3) a transmitter in the prior map.

In the first case, the new transmitter needs to be initialized with a high uncertainty. In the second case, the transmitter is initialized with the previously estimated state PDF. In the third case, the transmitter is initialized with the state PDF from the corresponding transmitter in the prior map. The question for which of the above three cases to decide is referred to as data association [9]. We use the data association scheme proposed in [10]. Every particle in the user particle filter decides on its own for associations. Hence, it is a multiple hypothesis data association scheme.

\section{Map Merging}

After a user has gone along its trajectory, a map is created to be passed on to the next user. In this map, the transmitter state PDFs estimated by the user and in the prior map have to be merged into one map. Since each user particle decides on associations among transmitters on its own, each particle has a belief whether a transmitter has or has not been associated, and therefore doesn't or does exist.

$\mathcal{T}_{j}$ is the set of user particle indices for which transmitter $j$ has not been associated with another transmitter. Thus, it does not contain the indices of particles who have decided for an association of transmitter $j$ with another transmitter. Marginalizing over the user state results in the posterior distribution for the $j^{\text {th }}$ transmitter

$$
\mathrm{p}\left(\boldsymbol{x}_{\mathrm{TX}, k}^{<\cdot, j>} \mid \boldsymbol{z}_{1: k}\right)=\sum_{i \in \mathcal{T}_{j}} w_{k}^{<i>} \sum_{l=1}^{N_{p, \mathrm{TX}}} w_{k}^{<i, j, l>} \delta\left(\boldsymbol{x}_{\mathrm{TX}, k}^{<\cdot, j>}-\boldsymbol{x}_{\mathrm{TX}, k}^{<i, j, l>}\right) .
$$

In order to reduce the storage and communication complexity, the particles for each transmitter are resampled with a particle reduction method similar to [11]. The transmitter state space is split into a discrete grid of bins. In each bin, only a certain number of particles is allowed. If more particles are to be resampled into a full bin, the particle is discarded and the weight of the particles in the bin is increased accordingly.

Since some or all of the user particles may have decided for an association among transmitter $j$ and another transmitter, each transmitter is assigned a likelihood of existence $\mathrm{p}_{e, j}$. This is the summed weight of particles that have not associated transmitter $j$ with any other transmitter, i.e.,

$$
\mathrm{p}_{e, j}=\sum_{i \in \mathcal{T}_{j}} w_{k}^{<i>}
$$

In the map, this likelihood is stored in addition to the particles and weights representing the state PDF for each transmitter. When another user receives this map as a prior map, association likelihoods with transmitters from the prior map are weighted with their likelihoods of existence $\mathrm{p}_{e, j}$.

\section{EVALUATIONS}

We evaluate our algorithm by simulations in an indoor scenario. Fig. 4 shows a top view of an indoor mall with one physical transmitter denoted by the red triangle labeled Tx. The physical transmitter continuously broadcasts a known signal of $100 \mathrm{MHz}$ bandwidth. The thick black lines are walls that reflect the transmit signal. The black dots are point scatterers.

For the simulations, the channel impulse response (CIR) is constructed at the user by ray-tracing for every user position. We assume a maximum of two interactions of the transmit signal before it arrives at the user, i.e., single and double reflections and/or scattering. The CIR is band-limited to $100 \mathrm{MHz}$, and white Gaussian noise (WGN) is added to obtain the received signal.

There are five users in the scenario, each of them walking through the scenario on its own track. The tracks for the single users are plotted in Fig. 4 in different colors. The $i^{\text {th }}$ user walks along the $i^{\text {th }}$ track whose starting point is labeled $\mathrm{S}_{i}$ and whose final point is labeled $\mathrm{E}_{i}$. All users move with a constant speed of $1 \mathrm{~m} / \mathrm{s}$.

Each user is equipped with a two-dimensional antenna array of nine elements. Hence, KEST can estimate both the ToAs and AoAs of the received signal components. An estimate from KEST is obtained every $50 \mathrm{~ms}$.

To evaluate our algorithm, we define the user walking along the blue track from $\mathrm{S}_{0}$ to $\mathrm{E}_{0}$ in Fig. 4 as reference user. We compare the MAE of the reference user for two cases: In the first case, the reference user walks along its track without any 


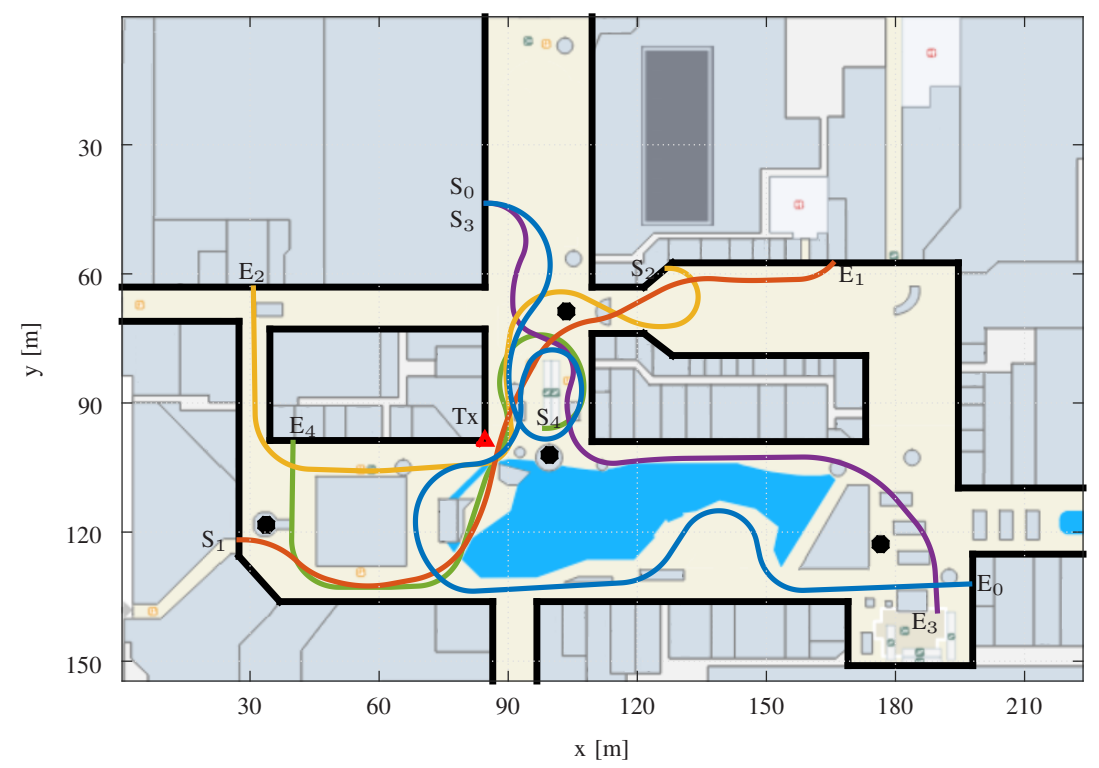

Fig. 4. The top view on the indoor simulation scenario with five different user tracks. The starting and end point of the $i^{\text {th }}$ user track are labeled $S_{i}$ and $\mathrm{E}_{i}$, respectively. The red triangle labeled $\mathrm{Tx}$ is placed at the physical transmitter location.

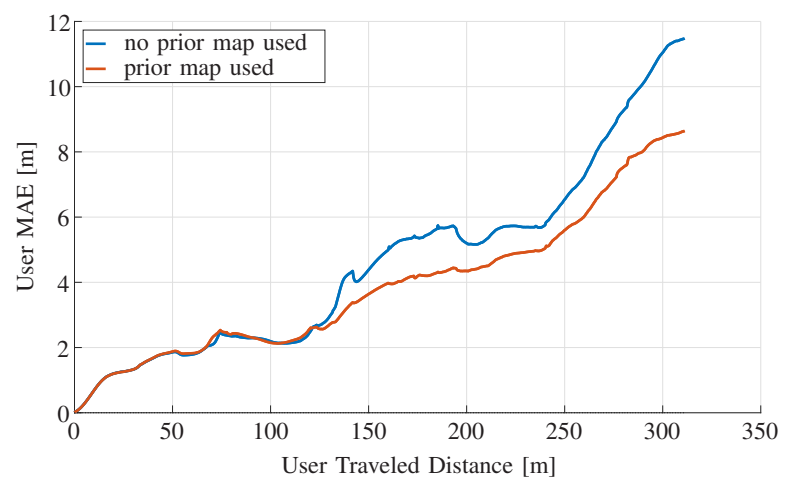

Fig. 5. The MAE of user 0 versus the traveled distance. No prior map is used for the curve in blue, whereas a prior map created and improved by the users 1 to 5 is used for the curve in red.

prior information on the locations of physical and virtual transmitters. For the second case, the reference user obtains a map of transmitter locations obtained by crowd-sourcing from the other four users as follows.

The first user walks along track 1 without prior knowledge on the transmitter locations, mapping the transmitters and creating a prior map of transmitters for the second user. The second user walks along track 2 exploiting the prior information on the transmitter states in the map obtained from the first user when a map match has been found. Having finished the walk along its trajectory, the second user updates the obtained map with its own observations and passes it on to the third user, and so on. Generally, the $i^{\text {th }}$ user obtains a prior transmitter map from the $(i-1)^{\text {th }}$ user, estimates the rotation and translation parameters relating the coordinate systems of the user and the obtained prior map, exploits the information in the map while walking along its track, updates the map with its own estimates and passes the map on to the $(i+1)^{\text {th }}$ user.

Finally, the reference user walks along the reference track 0, and exploits the information on the transmitters in the map after map matching.

The results of the simulations in terms of positioning error of the reference user for two cases are plotted in Fig. 5, where the MAE is plotted versus the traveled distance of the reference user. The red curve shows the MAE without any prior knowledge on transmitters. The blue curve denotes the MAE of the reference user with a prior map that has been created by the other four users as described above. The simulation results have been averaged over 110 runs for all users. 


\section{CONCLUSION}

In our multipath assisted positioning approach named Channel-SLAM, users create maps of physical and virtual transmitters in their own local coordinate systems. This paper shows how maps of physical and virtual transmitters can be shared and improved in a crowdsourcing approach. Users profit from prior maps in terms of a better positioning performance, and provide their updated and improved maps to other users.

The possibility of sharing maps turns Channel-SLAM from an ego-localization scheme to a cooperative approach, where cooperation means sharing maps among users and improving them. In addition, if a prior map has a known relation to a global geographic coordinate system, the user location can be determined on a global scale, turning Channel-SLAM from a relative to a global localization system.

\section{ACKNOWLEDGement}

This work was partially supported by the DLR project Navigation 4.0.

\section{REFERENCES}

[1] C. Gentner, T. Jost, W. Wang, S. Zhang, A. Dammann, and U.-C. Fiebig, "Multipath Assisted Positioning with Simultaneous Localization and Mapping," IEEE Transactions on Wireless Communications, vol. 15, no. 9, pp. 6104-6117, Sep. 2016.

[2] P. Setlur, G. Smith, F. Ahmad, and M. Amin, "Target Localization with a Single Sensor via Multipath Exploitation," IEEE Transactions on Aerospace and Electronic Systems, vol. 48, no. 3, pp. 1996-2014, Jul. 2012.

[3] P. Meissner and K. Witrisal, "Multipath-assisted single-anchor indoor localization in an office environment," in Systems, Signals and Image Processing (IWSSIP), 2012 19th International Conference on, Apr. 2012, pp. 22-25.

[4] H. Durrant-Whyte and T. Bailey, "Simultaneous localization and mapping: part I," IEEE Robotics and Automation Magazine, vol. 13, no. 2, pp. 99-110, Jun. 2006.

[5] M. Ulmschneider, D. C. Luz, and C. Gentner, "Exchanging Transmitter Maps in Multipath Assisted Positioning," in IEEE/ION Position, Location and Navigation Symposium (PLANS), 2018.

[6] T. Jost, W. Wang, U. Fiebig, and F. Perez-Fontan, "Detection and Tracking of Mobile Propagation Channel Paths," IEEE Transactions on Antennas and Propagation, vol. 60, no. 10, pp. 4875-4883, Oct. 2012.

[7] S. Kay, Fundamentals of Statistical Signal Processing: Estimation Theory, ser. Fundamentals of Statistical Signal Processing. Prentice-Hall PTR, 1998.

[8] A. Doucet, S. Godsill, and C. Andrieu, "On sequential Monte Carlo sampling methods for Bayesian filtering," Statistics and Computing, vol. 10, no. 3, pp. 197-208, Jul. 2000

[9] T. Bailey and H. Durrant-Whyte, "Simultaneous localization and mapping (SLAM): part II," IEEE Robotics and Automation Magazine, vol. 13, no. 3 , pp. 108-117, Sep. 2006.

[10] M. Ulmschneider, C. Gentner, T. Jost, and A. Dammann, "Multiple Hypothesis Data Association for Multipath-Assisted Positioning," in 14th Workshop on Positioning, Navigation and Communications (WPNC), Oct. 2017.

[11] C. Gentner, R. Phlmann, M. Ulmschneider, T. Jost, and S. Zhang, "Positioning Using Terrestrial Multipath Signals and Inertial Sensors," Mobile Information Systems, vol. 2017, 2017. 